\title{
Internationale Migration und (räumliche) Integration - Zeit für neues Denken
}

\author{
Ludger Pries
}

Online publiziert: 27. Juni 2013

(C) Springer-Verlag Berlin Heidelberg 2013

Die Stichwörter Migration und Integration umschreiben ein immer wichtiger werdendes Themenbündel und Diskursfeld - in Deutschland genauso wie in den meisten Ländern der Welt. Seit der verstärkten Globalisierung der 1980er Jahre gibt es kaum noch Länder, die nicht massive Aus- oder Einwanderungen erleben. Ganze Volkswirtschaften - wie z. B. die Tadschikistans oder Moldawiens - hängen von Geldrücküberweisungen von Migrierenden ab. Die Krisenherde der Welt - ob Afghanistan, Haiti, Irak, Somalia oder Syrien - produzieren massenhafte Flüchtlingswanderungen. Spanische und italienische Hochqualifizierte suchen angesichts der schlechten Wirtschaftslage ihrer Länder Erwerbsmöglichkeiten in Berlin oder Amsterdam. Die komplizierten Fragen des Umgangs mit Flüchtlingen und anderen Migrierenden - wie z. B. der sogenannten neuen Armutsmigration aus Rumänien und Bulgarien in Deutschland - werden teilweise für populistische Stimmungsmache und politische Profilierungssucht missbraucht.

Seit dem neuen Jahrhundert gerät das Themenfeld Migration und Integration in Deutschland aus einem dominanten Defizitfokus heraus. Demographische Entwicklungen und auch in Teilbereichen bereits spürbare Arbeitsmarktengpässe und andere Faktoren haben ein Umdenken eingeleitet. Die Chancen einer für Migration offenen Gesellschaft, die Potenziale der Migrierenden im Ankunftsland und auch die Chancen einer stärkeren Vernetzung mit den Herkunftsländern der Migrierenden werden zunehmend erkannt. Zudem kann das Thema Migration und Integration angesichts von EU-Freizügigkeit, substantiellen EU-Erweiterungen sowie EU-weiten Debatten um Gleichbehandlung

Prof. Dr. L. Pries $(\square)$

Fakultät für Sozialwissenschaft, Ruhr-Universität Bochum,

Universitätsstraße 150, 44789 Bochum, Deutschland

E-Mail: ludger.pries@rub.de und die Verknüpfung von Migration und Entwicklung immer weniger aus einer rein nationalgesellschaftlichen Perspektive bearbeitet werden. Integration als (Aushandlung über die) möglichst chancengleiche Teilhabe aller Menschen an den gesellschaftlich relevanten Lebensbereichen ist ein Prozess und gilt für Menschen mit wie ohne Migrationshintergrund, für solche mit deutscher, EU- oder Drittstaatsangehörigkeit.

Wie aber soll Integration dann im Einzelnen verstanden werden? Wie kann ein offenes, pluralistisches und aktivierendes Integrationskonzept weiterentwickelt werden, wenn sich die Migrations- und Mobilitätsformen (von Studierendenaustausch über Pendel- und Kettenwanderungen bis zu transnationaler Fachkräftemobilität innerhalb von Unternehmen) ausdifferenzieren und vermengen? Vor welchen Herausforderungen stehen Forschung und Politik?

Am Beispiel von zwei grenznahen Gemeinden - Perl im Saarland und Löcknitz-Penkun in Mecklenburg-Vorpommern - zeigen Birte Nienaber und Agnes Kriszan, dass nationalstaatliche Grenzen innerhalb der Europäischen Union weiterhin von Bedeutung sind, auch, weil und indem sie transnationale Lebenswelten hervorbringen. In den untersuchten Grenzregionen lebt ein nicht unerheblicher Teil der Bevölkerung in transnationalen Sozialräumen, indem z. B. die relevanten Lebensaktivitäten Arbeiten, Wohnen und Einkaufen in der gleichen Region, aber in verschiedenen Ländern vollzogen werden. Regionale Subzentren und eine gewisse Re-Regionalisierung können auch Ergebnis transnationaler Sozialräume sein. Raumplanung sollte zukünftig solche transnationalen Phänomene verstärkt berücksichtigen.

Die Besonderheiten der östlichen (neuen) Bundesländer Deutschlands - dünnbesiedelte Flächenstaaten mit starker allgemeiner Bevölkerungsfluktuation und niedrigen Migrierendenanteilen - werden von Sybille Münch behandelt. 
Nicht die in den alten Bundesländern klassische ,Gastarbeiter'-Migration bestimmt hier das Bild, sondern eine neue Zuwanderung seit den 1990er Jahren, vor allem durch (Spät-)Aussiedler, Flüchtlinge und Asylbeantragende sowie jüdische Kontingentflüchtlinge. Diese Gruppen von Migrierenden haben teilweise einen prekären Aufenthaltsstatus oder/und besondere Probleme auf dem Arbeitsmarkt. Westwärtswanderungen alteingesessener Bevölkerungsgruppen und neue (Pendel-)Migrationsformen auch mit Polen bestimmen das Bild ebenso wie stärkere Vorbehalte der Mehrheitsbevölkerung gegenüber Ausländern. Auch hier zeigt sich: Die spezifischen Herausforderungen von Integration sind nicht losgelöst von den besonderen Migrationstypen und -formen zu bearbeiten.

Heike Hanhörster und Christine Barwick untersuchen am Beispiel Duisburg und Berlin, aus welchen Gründen Türkeistämmige nicht nach bzw. mit ihrer wirtschaftlichen Etablierung aus ihrem angestammten benachteiligten Stadtviertel - im Sinne einer sozialräumlichen Entmischung - wegziehen. Ressourcenstarke Haushalte verbleiben in prekären Stadtteilen, weil sie durch Grenzziehungen innerhalb derselben und durch Netzwerkbildungen über deren Grenzen hinweg ihre soziale Lebenswelt gleichsam pluri-lokal - und manchmal auch transnational - gestalten können. Räumliche und soziale Nähe zur Familie und die lokal-räumliche Konzentration eines Großteils der sozialen Netzwerke erklären den Nicht-Wegzug. Auch hier zeigt sich wieder die enge Verquickung von Integration und (lokaler bzw. intranationaler Nicht-) Migration.

Henning Nuissl und Antonie Schmiz setzen an dem bereits erwähnten allmählichen Übergang von einer Defizitzu einer Potenzialperspektive hinsichtlich Migration an. Auf der Basis einer Analyse der internetbasierten Angebote von über 100 Gemeinden und Landkreisen aus allen Bundesländern zu den wirtschaftlichen Aktivitäten von Ausländern bzw. Menschen mit Migrationshintergrund kommen sie zu dem Schluss, dass eine solche potenzialorientierte und fördernde Perspektive allenfalls in Großstädten zu finden ist. Ansonsten herrscht eine defizitorientierte Behandlung von migrationsspezifischen Fragen in den Internetauftritten vor: (unterstützende) Bildungs- und Sozialpolitik, nicht potenzialorientierte Wirtschaftsförderung oder Stadtentwicklung werden im Zusammenhang mit migrantischer Selbstständigkeit vorrangig behandelt.

Handlungsbedarf für kommunale Raum- und Stadtentwicklungsplanung lässt sich auch aus dem Beitrag von Ariana Fürst und Jan Balke ableiten. Sie analysieren für Duisburg-Marxloh transnationale Unternehmensaktivitäten von Türkeistämmigen. Dieses transnationale Unternehmertum hat für die lokale Ökonomie und die Stadtteilentwicklung durchaus erhebliche, allerdings nicht immer angemessen berücksichtigte Relevanz. Die potenzielle ökonomische und soziale Stabilisierung von Stadtteilen durch ethnisches Unternehmertum kontrastiert dabei mit einer eher schwachen Netzwerkbildung und Integration solcher türkeistämmigen Unternehmungen.

Aus einer gänzlich anderen Perspektive wird das Thema multilokaler transnationaler Lebensweisen in dem Beitrag von Veronika Deffner und Carmella Pfaffenbach beleuchtet. Sie zeigen auf Basis von Literaturquellen am Beispiel der Hauptstadt des Sultanats Oman, was für die meisten Länder der Golfregion in ähnlicher Weise gilt: ,Zirkuläre' Migration von hunderttausenden Migrantinnen und Migranten vor allem aus Asien und Afrika ist inzwischen ein unverzichtbarer Teil des Funktionierens von Haushalten, Unternehmen und Staaten. Ähnlich wie in der alten europäischen ,Gastarbeiter'-Phase wird dabei eine dauerhafte Integration der Arbeitsmigrierenden von den Ankunftsländern nicht angestrebt. Über historisch verankerte soziale Institutionen wie das paternalistische Kafala-System (ein Haushaltsvorstand ,bürgt' für befristet eingestellte ausländische Arbeitskräfte und übernimmt die aufenthaltsrechtliche Verantwortung) kann dabei eine solche gewünschte Zirkulation von Arbeitskräften teilweise aufrechterhalten werden.

Der Beitrag von Andrea Dittrich-Wesbuer und Jörg Plöger könnte als eine Art resümierender Zusammenschau gelesen werden. Auf der Grundlage einer kritischen Literaturdurchsicht wird konstatiert, dass multilokale und transnationale Sozialräume und Lebensweisen heute das haushaltliche, kommunale und gesellschaftliche Leben wesentlich mitprägen - und zwar nicht nur im Zusammenhang von Migration, sondern auch etwa durch das pluri-lokale Aufwachsen von Kindern nach Trennung der Eltern. In der wissenschaftlichen Forschung und praktischen Diskussion zu Stadt- und Regionalentwicklung, aber auch zum Verständnis von Migration und Integration, von Wohnen und zivilgesellschaftlichem Engagement besteht zukünftig noch erheblicher Reflexions- und Handlungsbedarf. Dieses Schwerpunktheft kann wichtige Impulse für eine solche erweiterte Perspektive und Debatte geben.

Aus den Beiträgen zu diesem Schwerpunktheft und der wissenschaftlichen Diskussion um Migration und Integration ergeben sich fünf wesentliche Impulse für ein offenes, pluralistisches und aktivierendes Denken, welches auch für Stadtentwicklung bedeutsam ist. Erstens ist Integration keine ,Einbahnstraße' und kein ,Treppchen'. Sie folgt nicht einer festgelegten, stufenförmigen Sequenz, sondern ist ein ergebnisoffener Prozess. Zweitens ist Integration kein Mehrheitsdiktat, sie kann keine ,Anpassungskeule' gegen ,Andersartige' sein, vielmehr ist sie eine Verhandlungssache um die Teilhabe aller Gruppen eines Sozialgeflechtes. Das betrifft Deutsche und Nicht-Deutsche, Migrierende und Sesshafte, Reiche und Arme, Frauen und Männer. Drittens ist Integration nicht eine ,Entweder-oder'-Entscheidung, sondern eine ,Sowohl-als-auch'-Einladung bezüglich Loyalitäten, Heimatgefühlen, identitären Verortungen und 
Lebensperspektiven. Viertens ist Integration kein eindimensionaler Zustand, sondern ein permanenter und vieldimensionaler Prozess der ökonomischen, kulturellen, politischen und sozialen Teilhabe im Sinne einer aktiven und aktivierenden ,Mobilisierung von Fähigkeiten“. Schließlich ist Integration fünftens nicht notwendigerweise eine nur unilokale Fixierung im Sinne, russischer Puppen'. Menschen sind zunehmend transnational mobil, sie können sich unabhängig von ihrem Wohnort auch mit dem eigenen oder dem Herkunftsland ihrer Eltern oder Großeltern verbunden fühlen. Zunehmende transnationale Mobilität führt auch zu einer pluri-lokalen Einbindung auf lokaler, nationaler, supranationaler, globaler und transnationaler Ebene. Je stärker eine offene Gesellschaft plurale Formen der aktiven und aktivierenden Teilhabe ermöglicht, desto attraktiver wird sie auf Dauer für Menschen unterschiedlicher Staatsangehörigkeiten, die diese Werte als Referenzrahmen teilen. 